\title{
Origin of magnetic switching field distribution in bit patterned media based on pre-patterned substrates
}

Cite as: Appl. Phys. Lett. 99, 062502 (2011); https://doi.org/10.1063/1.3623488

Submitted: 16 April 2011 • Accepted: 19 July 2011 • Published Online: 08 August 2011

B. Pfau, C. M. Günther, E. Guehrs, et al.

ARTICLES YOU MAY BE INTERESTED IN

Role of reversal incoherency in reducing switching field and switching field distribution of exchange coupled composite bit patterned media

Applied Physics Letters 95, 262504 (2009); https://doi.org/10.1063/1.3276911

Bit patterned media based on block copolymer directed assembly with narrow magnetic switching field distribution

Applied Physics Letters 96, 052511 (2010); https://doi.org/10.1063/1.3293301

Influence of ion irradiation on switching field and switching field distribution in arrays of Co/

Pd-based bit pattern media

Applied Physics Letters 98, 172506 (2011); https://doi.org/10.1063/1.3581896

母QBLOX

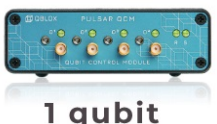

Shorten Setup Time Auto-Calibration More Qubits

Fully-integrated Quantum Control Stacks Ultrastable DC to $18.5 \mathrm{GHz}$ Synchronized $<<1$ ns Ultralow noise

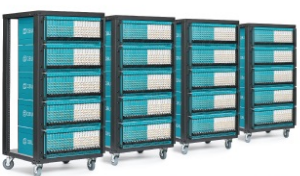

100s qubits

visit our website > 


\title{
Origin of magnetic switching field distribution in bit patterned media based on pre-patterned substrates
}

\author{
B. Pfau, ${ }^{1,2}$ C. M. Günther, ${ }^{1,2}$ E. Guehrs, ${ }^{1}$ T. Hauet, ${ }^{3, a)}$ H. Yang,,${ }^{3, b}$ L. Vinh, ${ }^{3}$ X. Xu, ${ }^{3}$ \\ D. Yaney, ${ }^{3}$ R. Rick, ${ }^{4}$ S. Eisebitt, ${ }^{1,2}$ and O. Hellwig ${ }^{3, c)}$ \\ ${ }^{1}$ TU Berlin, Institut für Optik und Atomare Physik, Hardenbergstraße 36, 10623 Berlin, Germany \\ ${ }^{2}$ Helmholtz-Zentrum Berlin für Materialien und Energie GmbH, Albert-Einstein-Str. 15, 12489 Berlin, \\ Germany \\ ${ }^{3}$ San Jose Research Center, Hitachi Global Storage Technologies, 3403 Yerba Buena Rd., San Jose, \\ California 95135, USA \\ ${ }^{4}$ Stanford Synchrotron Radiation Laboratory, SLAC, Menlo Park, California 94025, USA
}

(Received 16 April 2011; accepted 19 July 2011; published online 8 August 2011)

\begin{abstract}
Using a combination of synchrotron radiation based magnetic imaging and high-resolution transmission electron microscopy we reveal systematic correlations between the magnetic switching field and the internal nanoscale structure of individual islands in bit patterned media fabricated by $\mathrm{Co} / \mathrm{Pd}$-multilayer deposition onto pre-patterned substrates. We find that misaligned grains at the island periphery are a common feature independent of the island switching field, while irregular island shapes and misaligned grains specifically extending into the center of an island are systematically correlated with a reduced island reversal field. (C) 2011 American Institute of Physics. [doi:10.1063/1.3623488]
\end{abstract}

Bit patterned media (BPM) is a leading candidate for extending high-density magnetic recording in hard disk drives beyond $1 \mathrm{~Tb} / \mathrm{in}^{2}$. BPM technology aims at increasing the recording density by pre-defining the bits, i.e., the magnetic volume storing one information unit, as lithographically separated islands. ${ }^{1}$ The bit-to-bit variation of the coercive fields is expressed in the switching field distribution (SFD). In order to reliably write a bit, i.e., to reverse the magnetization of an island, the SFD of the island array has to be sufficiently narrow. Currently the controlled reduction of the SFD remains a major challenge in the development of BPM. The SFD is determined by two different contributions. ${ }^{2}$ First the magnetic islands are influenced by the stray fields of neighboring islands. In case of perpendicular anisotropy systems that are especially considered for applications in BPM the stray fields from neighbors tend to broaden the SFD. Second the switching field of every single island is influenced by its individual properties. Due to island-to-island variations of these properties, the switching fields show a so-called intrinsic distribution. Variations in island reversal fields are assumed to be induced by variations of the magnitude or direction of the local anisotropy, such as originating from island size, shape, composition, and (crystalline) structure as well as localized strain, corrupted island edges, or misaligned grains. ${ }^{3-7}$

In a recent study, Lau et al. found that islands with a low switching field (easy switchers) do predominantly contain a "trigger" grain larger than the exchange length with in-plane [100] vector, whereas islands with high switching field (stubborn or hard switchers) do not. ${ }^{7}$ The magnetic nanodots in Ref. 7 were fabricated by transferring an island array hard

\footnotetext{
${ }^{a)}$ Present address: Institut Jean Lamour, Nancy-Université, CNRS, boulevard des aiguillettes, BP 329, F-54506 Vandoeuvre lès Nancy, France.

b) Present address: Seagate Technology, 47010 Kato Rd., Fremont, California 94538, USA.

${ }^{c}$ Electronic mail: Olav.Hellwig@hitachigst.com.
}

mask defined via electron-beam lithography into a continuous $\mathrm{Co} / \mathrm{Pd}$ multilayer film using Ar sputter etching. Here it is expected that misaligned grains are uniformly distributed among and within the islands. This could be different for BPM based on pre-patterned substrates where the magnetic islands form on top of cuboidal pillars. ${ }^{8}$ The scheme of our experiment is similar to Ref. 7. First, the easy and hard switchers in a set of islands are identified by imaging the islands' magnetization state in a complete hysteresis loop. Then, the shape and the crystalline structure of a subset of particular nanodots are analyzed via high-resolution transmission electron microscopy (TEM) and fast Fourier transforms (FFTs) generated from high-resolution TEM images of individual islands. In contrast to the previous study, we use the $\mathrm{x}-$ ray Fourier transform holography (FTH) $\operatorname{method}^{9}$ for magnetic imaging and increase the statistics in the hysteresis loop, in order to better identify systematic easy and hard switchers.

The sample was prepared by sputter depositing a $\mathrm{Co} / \mathrm{Pd}$ multilayer $\left(\mathrm{Ta}(15) / \mathrm{Pd}(30) /[\mathrm{Co}(5.5) / \mathrm{Pd}(9)]_{24} / \operatorname{Pd}(11) \AA\right)$ onto a pre-patterned, $150 \mathrm{~nm}$ thick $\mathrm{Si}_{3} \mathrm{~N}_{4}$ membrane window (Fig. 1(a)). The magnetic model system was chosen to fit the experimental pre-conditions in terms of available saturation field and necessary imaging contrast. The leading perpendicular anisotropy term in $\mathrm{Co} / \mathrm{Pd}$ multilayers originates from the $\mathrm{Co} / \mathrm{Pd}$ interfaces. Furthermore the $\mathrm{Ta} / \mathrm{Pd}$ seed layers provide an outof-plane FCC [111] preferred grain crystal orientation in the Pd-rich multilayer with an $\mathrm{x}$-ray rocking curve full width at half maximum of $7^{\circ}-8^{\circ}$ that creates an additional second source of perpendicular anisotropy. ${ }^{10}$ The patterned areas are $(6 \times 6) \mu \mathrm{m}^{2}$ in size and consist of a periodic array of cuboidal pillars of size $(80 \times 80 \times 40) \mathrm{nm}^{3}$ produced by e-beam lithography using polymethyl methacrylate resist and a CrMo (20 $\mathrm{nm}$ ) alloy etch mask. The membrane window was generated by a potassium hydroxide silicon wet etch to remove the bulk of the silicon from underneath the membrane. The dot period is $120 \mathrm{~nm}$, corresponding to an island area filling factor of 

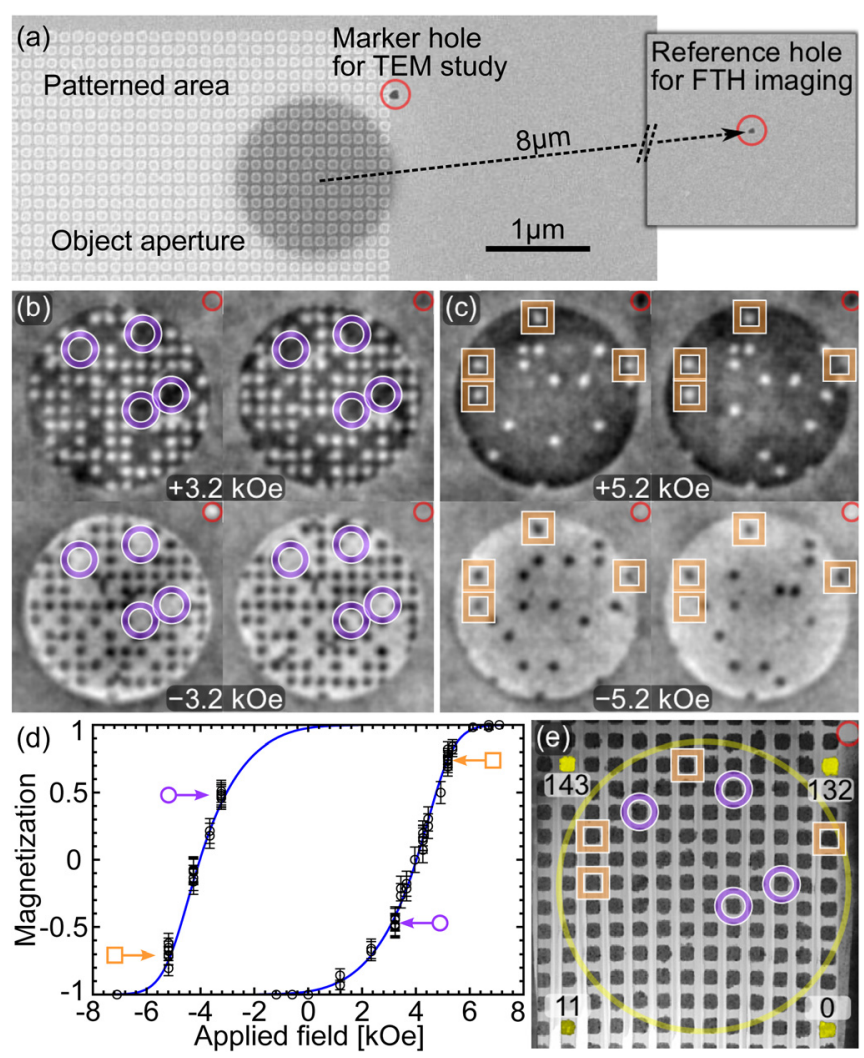

FIG. 1. (Color online) (a) Scanning electron microscope image of the integrated sample design. (b), (c) Examples of FTH magnetization state images at different applied fields. (d) Hysteresis loop for the dot ensemble in the FOV compiled out of 60 images. On the rising branch of the loop the islands switch from white to black, and vice versa. Images (b) are taken when the reversal is initiated and images (c) just before it is completed. The four easy (violet circles) and the four hard (orange squares) switchers that are selected for further TEM analysis are marked in the FTH images and in the TEM overview image (e) with the help of the marker hole (red). In (e) the yellow circle marks the holography FOV and the numbers illustrate the island labeling.

$44 \%$ and a bit density of $41 \mathrm{Gbit} / \mathrm{in}^{2}$. On the opposite side of the membrane, an FTH optical mask is produced into a $1 \mu \mathrm{m}$ thick gold layer via focused ion beam (FIB) lithography. The mask contains an object aperture with a diameter of $1.5 \mu \mathrm{m}$ defining the field of view (FOV) and a $50 \mathrm{~nm}$ wide reference hole needed for the image reconstruction. ${ }^{9}$ In addition, a small marker hole was drilled next to the FOV to attribute identical dots in the FTH and TEM images.

The FTH experiments were performed at the undulator beamline UE52-SGM of the BESSY II synchrotron source in Berlin, Germany. The energy of the circularly polarized photons was tuned to the $\mathrm{Co}_{3}$ edge $(778.1 \mathrm{eV})$ giving rise to a pronounced X-ray magnetic circular dichroism (XMCD) contrast in the images, which is further enhanced by subtracting all non-magnetic contributions. In the purely magnetic contrast images (Figs. 1(b) and 1(c)), the dots which have not switched appear on the uniform background of the already reversed continuous film in the trenches. Since the FTH method allows imaging the sample in an applied field, we were able to compare the island magnetization in applied field and remanence (not shown). As can be expected, we never observed a re-reversal of an island when turning off the external field. Nevertheless, all results presented throughout this paper are obtained from images taken in applied field.
In total $N=112$ dots are visible in the FTH FOV. They are labeled in a $(12 \times 12)$ elements matrix starting with 0 in the right bottom corner and ending with 143 in the left top corner (Fig. 1(e)). By counting the number of reversed islands $(n)$ at a certain applied field $(H)$ we gain a complete hysteresis loop for the dot ensemble in our FOV (Fig. 1(d)). The normalized magnetization $(M)$ of the dot ensemble is calculated as $2 n / N-1$, the statistical error bars as $2 / N$ $(n(1-n / N))^{0.5}$ (hypergeometric distribution). Altogether, we recorded 60 images at 22 different field values. Following Refs. 2 and 11, the results are fitted with an asymmetric error function, which is analytically defined as an inverse function $H(M)=H_{C}+\sqrt{2} \sigma \operatorname{erf}^{-1}(M) /(1+\alpha M)$ with $H_{\mathrm{C}}$ being the coercive field, $\sigma$ the width of the SFD, and $\alpha$ the asymmetry parameter. For our sample we obtain $H_{\mathrm{C}}=(4.02 \pm 0.04) \quad \mathrm{kOe}, \quad \sigma=(1.25 \pm 0.03) \mathrm{kOe}$ and $\alpha$ $=0.30 \pm 0.03$.

The hysteresis loop gives only information on the ensemble average switching behavior but does not reveal the individual properties of the dots, in particular the repeatability of the switching. For this analysis, we recorded ten independent configurations at field values of $H^{\text {easy }}= \pm 3.2 \mathrm{kOe}$ and $H^{\text {hard }}= \pm 5.2 \mathrm{kOe}$ (Figs. 1(b) and 1(c)) (after each time saturating the sample), where $25 \%$ and $87 \%$ of the dots have switched, respectively. In Fig. 2(a) detailed statistics for every island are presented showing how frequent each island reversed its magnetization. The statistics reveal three different groups of islands which are further summarized in a histogram in Fig. 3(a): (i) islands that always did not switch, (ii) islands that occasionally switched, and (iii) islands that always switched. The apparent coexistence of deterministic and nondeterministic processes is explained in a statistical broadening of the dot's individual switching field from the intrinsic value due to thermal activation and due to the local demagnetizing field from the dot's environment, which varies with the actual magnetic configuration. When applying a certain external field, the field is either much lower (island group (i)), or much higher than the individual intrinsic switching field (group (iii)), or lies within the statistical broadening of the individual intrinsic switching field resulting in the random behavior of group (ii). We have selected four islands belonging to group (iii) at a field value, where the switching is initiated ( $\left.H^{\text {easy }}\right)$ and four islands belonging to group (i) at the end of the reversal process $\left(H^{\text {hard }}\right)$ for a detailed TEM analysis in order to reveal the micro-structural peculiarities of the dots. We call those islands easy and hard switchers, respectively. As most of the selected islands show the same behavior in every run we find a probability of $99.9 \%$ (binomial significance test) that this behavior is not random (in the case of seven out of ten runs, the probability drops to $83 \%$ ). The positions of the selected islands in the dot array are also marked in the FTH images and the TEM overview image (Figs. 1(b), 1(c) and 1(e)).

A TEM sample was prepared from the array of nanodots (Fig. 1(e)) using a FIB milling technique, in which the space between the islands was initially filled by e-beam deposited Si-oxide. Following removal of a FIB chip, the sample was thinned to electron transparency by milling from the substrate side of the islands, leaving only islands and filler material in the final thin section. As shown in Fig. 2, highresolution TEM images were acquired from four easy (b) 


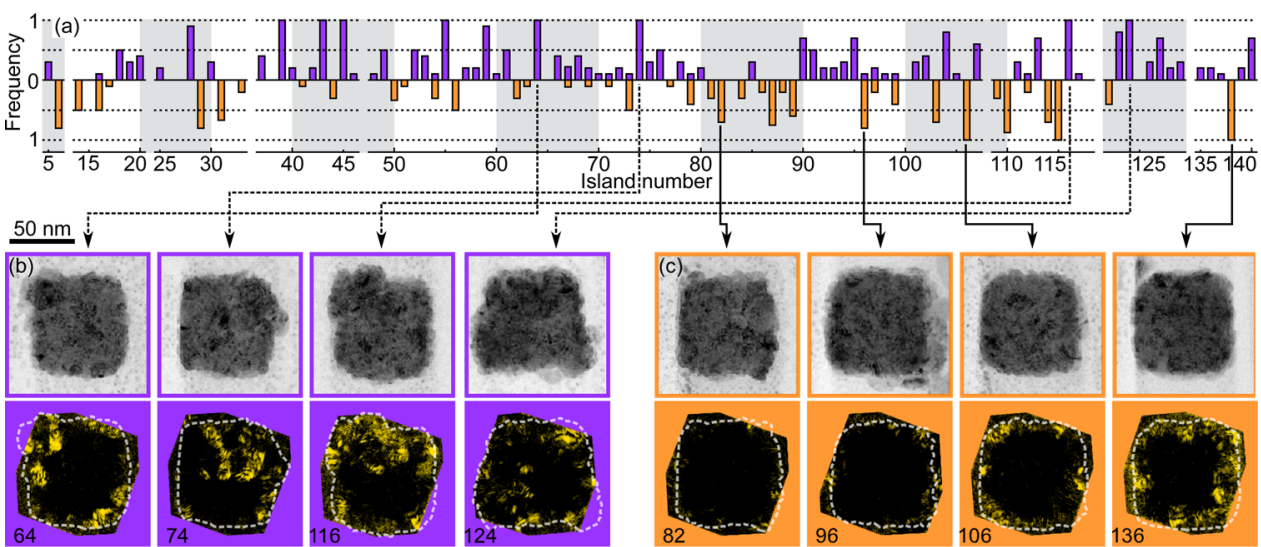

FIG. 2. (Color online) (a) Detailed statistics of how often each island is among the first (violet, up) or last (orange, down) islands that switch. Note that a few labeled dots in Fig. 1(e) are missing in the statistics since they do not appear in the circular FTH images. For the TEM analysis (b), (c) only islands with high probability for being either easy (b) or hard switchers (c) were selected. In the top row of (b) and (c) we show bright-field TEM images and in the bottom row corresponding inverse FFTs generated using only lattice spacing information ranging from 0.185 to $0.200 \mathrm{~nm}$. and four hard switching islands (c). FFTs of each of the high-resolution images were processed to remove information related to lattice spacing outside of the range of 0.185 to $0.200 \mathrm{~nm}$. In other words, only information related to the (200) lattice spacing (average value from TEM analysis was $0.191 \mathrm{~nm}$ ) was retained. Subsequently generated inverse FFTs show the island regions with in-plane (200) lattice spacing, i.e., grains that are significantly misaligned from the FCC [111] out-of-plane crystal orientation, which is the origin of the second source of perpendicular anisotropy. ${ }^{10}$

In agreement with Ref. 7 we identified (200) grains in every easy switcher. These grains are large enough to reduce the average anisotropy of an island significantly. However, we also find hard switchers containing (200) grains (islands no. 106 and 136). But in contrast to easy switchers, these grains are always located at the edges of the island. Obviously, the peripheral grains do not always act as trigger grains. This observation is in agreement with micromagnetic simulations, where the switching field of a nanodot depending on the position of a "magnetic defect" in the dot was determined. ${ }^{4}$ It was found that in some cases-depending on the tilt of the anisotropy-the switching field even increases if the defect is located at the edge of the island. Another explanation of our observations is the assumption that the islands have a low anisotropy shell at the edges which may be due to imperfections in the layering, surface anisotropy, or oxidation. In this case mis-oriented grains within the low anisotropy shell would have less impact on the SFD than grains located more towards the center of an island. In addition to the grain structure of the islands
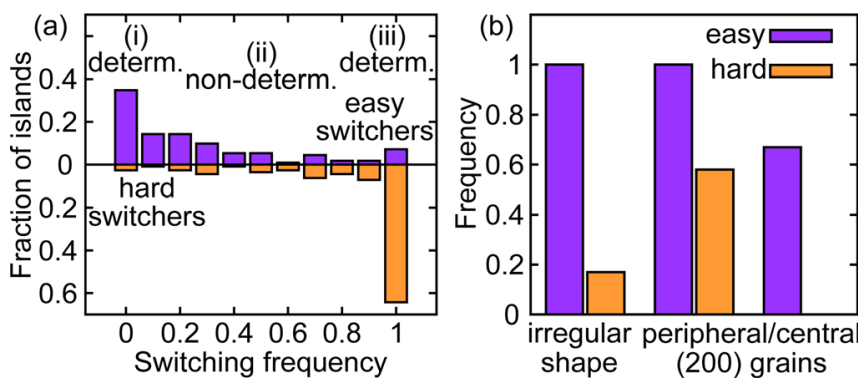

FIG. 3. (Color online) (a) Histogram of Fig. 2(a) at applied fields of $H^{\text {easy }}$ (violet, up) and $H^{\text {hard }}$ (orange, down). The easy (hard) switchers were selected from the small group of dots that have deterministically switched (not switched) at $H^{\text {easy }}\left(H^{\text {hard }}\right)$. (b) Appearance rate of selected features in easy and hard switchers as observed in TEM analysis. we also recognize that the hard switchers exhibit a more regular squared island shape without any significant deformations as found for easy switchers.

In order to enhance the significance of the statistics we included islands from other identical magnetic pattern arrays (not shown here) into the TEM study as well. In total we investigated 12 easy and 12 hard switchers with respect to central or peripheral (200) grains and irregularities in the overall island shape. The analysis presented in Fig. 3(b) reveals that most nanodots, even most hard switchers, have at least one (200) grain located at the periphery of the dot. But apparently these grains are not always able to initiate the magnetic reversal. Thus, we conclude that the absence of a central (200) grain is characteristic of hard switchers. In addition we find indications that the irregular island shape is correlated with the presence of (200) grains. This finding is not surprising as a disordered growth will favor various grain alignments and vice versa. Finally, we can conclude that for reducing the intrinsic SFD in BPM it is necessary to either avoid defects (mostly in the form of misaligned grains) or to create identical defects in each island. Another option may be realized in the form of exchange coupled composite (ECC) BPM structures, ${ }^{12}$ where the island reversal is initiated by a nucleation assist layer that can be separately optimized with respect to defects.

${ }^{1}$ T. R. Albrecht, O. Hellwig, R. Ruiz, M. E. Schabes, B. D. Terris, and X. Z. Wu, Nanoscale Magnetic Materials and Applications, edited by J. P. Liu, E. E. Fullerton, O. Gutfleisch, and D. J. Sellmyer (Springer Science + Business Media, New York, 2009), pp. 237-274.

${ }^{2}$ O. Hellwig, A. Berger, T. Thomson, E. Dobisz, Z. Z. Bandic, H. Yang, D. S. Kercher, and E. E. Fullerton, Appl. Phys. Lett. 90, 162516 (2007).

${ }^{3}$ T. Thomson, G. Hu, and B. D. Terris, Phys. Rev. Lett. 96, 257204 (2006).

${ }^{4}$ J. M. Shaw, M. Olsen, J. W. Lau, M. L. Schneider, T. J. Silva, O. Hellwig,

E. Dobisz, and B. D. Terris, Phys. Rev. B 82, 144437 (2010).

${ }^{5}$ G. Hu, T. Thomson, C. Rettner, and B. D. Terris, IEEE Trans. Magn. 41, 3589 (2005).

${ }^{6}$ R. Dittrich, G. Hu, T. Schrefl, T. Thomson, D. Suess, B. D. Terris, and J. Fidler, J. Appl. Phys. 97, 10J705 (2005).

${ }^{7}$ J. W. Lau, R. D. McMichael, S. H. Chung, J. O. Rantschler, V. Parekh, and D. Litvinov, Appl. Phys. Lett. 92, 012506 (2008).

${ }^{8}$ O. Hellwig, T. Hauet, T. Thomson, E. Dobisz, J. D. Risner-Jamtgaard, D. Yaney, B. D. Terris, and E. E. Fullerton, Appl. Phys. Lett. 95, 232505 (2009).

${ }^{9}$ S. Eisebitt, J. Lüning, W. F. Schlotter, M. Lörgen, O. Hellwig, W. Eberhardt, and J. G. Stöhr, Nature 432, 885 (2004).

${ }^{10}$ B. N. Engel, C. D. England, R. A. Van Leeuwen, M. H. Wiedmann, and C. M. Falco, Phys. Rev. Lett. 67, 1910 (1991).

${ }^{11}$ A. Berger, B. Lengsfield, and Y. Ikeda, J. Appl. Phys. 99, 08 E705 (2006).

${ }^{12}$ T. Hauet, E. Dobisz, S. Florez, J. Park, B. Lengsfield, B. D. Terris, and O. Hellwig, Appl. Phys. Lett. 95, 262504 (2009). 